\title{
Prévention des ulcères : pas de stress!
}

\author{
par Robert MacLaren
}

$\mathrm{U}$ ne théorie médicale répandue veut que les patients gravement malades nécessitent une prophylaxie de l'ulcère de stress (PUS) pour prévenir le saignement gastro-intestinal (SGI) engendré par une ischémie de la muqueuse à la suite d'un stress physiologique. Refuser la PUS à un patient présentant un risque de SGI ou la désamorcer peut être perçu comme une inconduite médicale ou un manquement au respect des mesures standard de rendement. La PUS est tellement ancrée dans la pratique que de nombreux services de soins intensifs (SSI) disposent de modèles d'ordonnances normalisés au moment de l'admission, qui stipulent d'entreprendre automatiquement la PUS. Malheureusement, la conséquence involontaire de son déclenchement irréfléchi dans les SSI résulte en la propagation de cette pratique aux patients ne présentant pas d'indication de PUS. Le taux en baisse du SGI ainsi que l'association entre la suppression de l'acide et les complications infectieuses sont sources de scepticisme à l'égard de la PUS. Il y a deux ans, Yamashita et Duffett plaidaient dans ce journal en faveur de la PUS et contre elle à l'occasion d'un débat où s'affrontaient le pour et le contre ${ }^{1,2}$. Mon objectif vise ici à mettre en évidence d'autres considérations, y compris certains résultats clés d'études récemment publiées, pour attirer l'attention sur le dilemme clinique persistant relatif à la PUS.

Les deux facteurs de risque de SGI lié au stress les plus communément cités sont la ventilation mécanique et la coagulopathie. Ces facteurs de risque sont mentionnés dans une étude observationnelle menée auprès de 2252 patients des SSI. Dans cette étude, les chercheurs ont demandé la suspension de la PUS, à moins que le patient soit atteint d'un traumatisme crânien, qu'il ait subi des brûlures thermiques importantes ou une greffe, qu'il ait souffert récemment d'un ulcère peptique ou d'un SGI; finalement, 674 patients ont reçu une PUS et 1578 n'en ont pas reçu ${ }^{3}$. La présence d'hypotension tendait à être significativement associée au SGI. L'indication principale, qui concernait 54,8 \% des patients, mentionnait une maladie cardiovasculaire ou une chirurgie, pour lesquelles les pratiques médicales avaient évolué principalement de l'anticoagulation et de la chirurgie aux techniques d'intervention radiologique non invasives. Peu de patients avaient reçu un diagnostic de lésion du système nerveux central, de septicémie, de traumatisme crânien ou de traumatismes multiples. La ventilation non invasive n'était pas systématiquement utilisée au moment de la publication de l'étude. Par conséquent, la lecture des résultats de cette étude doit s'inscrire dans le contexte de la population évaluée, de l'exclusion des patients présentant des facteurs de risque et des changements aux pratiques médicales qui ont eu lieu depuis leur publication. Revenons à aujourd'hui et à la publication récente d'une méta-analyse portant sur huit études (116497 patients), qui démontre que la coagulopathie, le choc et la maladie hépatique chronique sont associés à un SGI important sur le plan clinique, mais que la ventilation mécanique ne l'est pas ${ }^{4}$. Les personnes en faveur de la PUS noteront que la plupart des études incluses dans cette méta-analyse y ont eu recours; il faut donc considérer ces paramètres comme des facteurs de risque lors de l'administration de cette prophylaxie. Les adversaires de la PUS souligneront quant à eux le manque d'uniformité des études et ils voudront également savoir si les facteurs de risque "établis» sont véritablement connus.

Alors que les objectifs de la thérapie se concentrent sur la mortalité, sur le SGI cliniquement important et sur les complications infectieuses, on prescrit communément la PUS sans se préoccuper vraiment des avantages et des inconvénients de chaque agent. L'usage courant des antagonistes du récepteur $\mathrm{H} 2$ de l'histamine (H2RA) repose simplement sur une étude randomisée en double aveugle, qui a été menée auprès de 1200 patients ventilés mécaniquement; celle-ci a révélé un taux moins élevé de SGI cliniquement significatifs sous l'effet de la ranitidine plutôt que de la sucralfate $(1,7 \%$ contre 3,8\%, $p=0,02)^{5}$. Pourtant, une méta-analyse récente incluant cette étude décisive n'a trouvé aucune différence de SGI cliniquement importante en présence des H2RA et de la sucralfate, mais il y avait moins de pneumonie en présence de la sucralfate ${ }^{6}$. Notons que la plupart des études incluses impliquaient l'administration des H2RA par infusion ou adaptation posologique pour obtenir des valeurs de $\mathrm{pH}$ gastriques supérieures à 3,5-4. Ces deux aspects de l'administration du médicament peuvent modifier le microbiome gastro-intestinal, ce qui augmente le risque d'infection dans une plus large mesure que l'administration conventionnelle et intermittente de H2RA. Les résultats d'une récente méta-analyse n'indiquent une SGI inférieure avec des inhibiteurs de la pompe à protons (PPI) qu'avec des $\mathrm{H}_{2} \mathrm{RA}^{7}$; cependant, les résultats étaient influencés par deux études 
présentant des lacunes méthodologiques. En revanche, les analyses pharmacoépidémiologiques ont révélé des taux de pneumonie et d'infection de Clostridioides difficile moins importants en présence des H2RA; cela permet à nouveau de penser que le degré d'importance de la suppression d'acide contribue aux perturbations du microbiome ${ }^{8,9}$. Plus récemment, une étude randomisée en double aveugle et contrôlée par placebo a mis en évidence des taux plus faibles de SGI cliniquement importants en présence de pantoprazole $(2,5 \%$ contre $4,2 \%$, risque relatif 0,$58 ; 95 \%$ intervalle de confiance $0,4-0,86)^{10}$. Malgré la similitude des complications infectieuses et le résultat principal qui portait sur la mortalité 90 jours après le traitement, tous groupes confondus, une analyse post hoc a démontré des taux de mortalité plus élevés sous l'effet du pantoprazole parmi les patients les plus gravement malades (c.-à-d. ceux qui sont le plus exposés aux facteurs de risque de SGI ${ }^{11}$. L'ensemble de ces données compliquent le choix de la classe d'agents à privilégier pour entreprendre une PUS, ce qui met en évidence le besoin de définir quels résultats sont les plus importants. Malgré que le SGI soit associé à un séjour prolongé en SSI et à des coûts supplémentaires, aucune étude n’a démontré d'avantage sur la mortalité après une PUS. Le risque de complications infectieuses et les taux de mortalité plus élevés que l'analyse post hoc de l'étude la plus récente ne peut pas expliquer ${ }^{11}$ créent de l'incertitude entourant la pratique courante de la PUS.

Le déclin du SGI lié au stress au cours des dernières décennies s'explique en partie par l'efficacité supérieure des stratégies de PUS ou par des pratiques médicales contemporaines (comme la réanimation hémodynamique énergique) qui limitent l'ischémie de la muqueuse. L'administration précoce de nutrition entérale peut protéger contre le SGI dans la mesure où l'efficacité de la PUS pharmacologique diminue ${ }^{12}$. En dernier lieu, la tolérance à la nutrition entérale signifie que la reperfusion gastro-intestinale s'est déroulée, que les facteurs de risque de SGI demeurent présents ou non. La durée de la PUS a grandement diminué, puisque l'étude la plus récente propose environ quatre jours de thérapie, ce qui coïncide avec le moment où le SGI risque le plus de se produire après l'admission dans les SSI ${ }^{10}$. Malheureusement, la pratique actuelle ne reflète pas cette tendance, puisque $25 \%$ des patients continuent de recevoir inutilement une PUS après leur congé de l'hôpital. L'argument pour ou contre la PUS ne devrait pas se focaliser sur l'adoption ou l'abandon universel de la pratique, mais plutôt sur la manière de rationaliser sa bonne utilisation pour optimiser la prévention du SGI, tout en limitant l'exposition aux médicaments et en réduisant les conséquences négatives. Plutôt que de cesser la thérapie, les investigateurs proposent un modèle de pratique plus sûr, qui consiste à limiter les ordonnances de la PUS à deux ou trois jours, alors que la prolongation de la durée nécessiterait une nouvelle ordonnance du prescripteur. L'étude des facteurs de risque a révélé que le taux de SGI était considérablement plus élevé dans la cohorte ayant reçu une PUS (16,3\% contre $1,5 \%)^{3}$. Certains pourraient prétendre que ce résultat est un signe d'inefficacité de la PUS, alors qu'il reflète plutôt un biais de sélection : les cliniciens étant en effet plus enclins à administrer une PUS aux patients perçus comme présentant un risque plus élevé de SGI. Les études en cours et les nouvelles lignes directrices pourraient faire la lumière sur certaines incertitudes, mais entretemps, il est important de comprendre l'équilibre clinique entourant la PUS et d'assurer une thérapie appropriée en la matière, tout en dissipant la croyance selon laquelle elle serait un rite de passage obligatoire dans les SSI.

[Traduction par l'éditeur]

\section{Références}

1. Yamashita S. Should we continue stress ulcer prophyalxis for critically ill patients? The "pro" side. Can J Hosp Pharm. 2017;70(4):316-7.

2. Duffett M. Should we continue stress ulcer prophyalxis for critically ill patients? The "con" side. Can J Hosp Pharm. 2017;70(4):317-8.

3. Cook DJ, Fuller HD, Guyatt GH, Marshall JC, Leasa D, Hall R, et al.; Canadian Critical Care Trials Group. Risk factors for gastrointestinal bleeding in critically ill patients. $N$ Engl J Med. 1994;330(6):377-81.

4. Granholm A, Zeng L, Dionne JC, Perner A, Marker S, Krag M, et al. Predictors of gastrointestinal bleeding in adult ICU patients: a systematic review and meta-analysis. Intensive Care Med. 2019;45(10):1347-59.

5. Cook D, Guyatt G, Marshall J, Leasa D, Fuller H, Hall R, et al.; Canadian Critical Care Trials Group. A comparison of sucralfate and ranitidine for the prevention of upper gastrointestinal bleeding in patients requiring mechanical ventilation. $N$ Engl J Med. 1998;338(12):791-7.

6. Alquraini M, Alshamsi F, Møller MH, Belley-Cote E, Almenawer S, Jaeschke $\mathrm{R}$, et al. Sucralfate versus histamine 2 receptor antagonists for stress ulcer prophylaxis in adult critically ill patients: a meta-analysis and trial sequential analysis of randomized trials. J Crit Care. 2017;40:21-30.

7. Alshamsi F, Belley-Cote E, Cook D, Almenawer SA, Alqahtani Z, Perri D, et al. Efficacy and safety of proton pump inhibitors for stress ulcer prophylaxis in critically ill patients: a systematic review and meta-analysis of randomized trials. Crit Care. 2016;20(1):Article 120.

8. Krag M, Marker S, Perner A, Wetterslev J, Wise MP, Schefold JC, et al.; SUP-ICU Trial Group. Pantoprazole in patients at risk for gastrointestinal bleeding in the ICU. N Engl J Med. 2018;379(23):2199-208.

9. Marker S, Perner A, Wetterslev J, Krag M, Lange T, Wise MP, et al.; SUP-ICU Investigators. Pantoprazole prophylaxis in ICU patients with high severity of disease: a post hoc analysis of the placebo-controlled SUP-ICU trial. Intensive Care Med. 2019;45(5):609-18.

10. Lilly CM, Aljawadi M, Badawi O, Onukwugha E, Tom SE, Magder LS, et al. Comparative effectiveness of proton pump inhibitors vs histamine type 2 receptor blockers for preventing clinically important gastrointestinal bleeding during intensive care: a population-based study. Chest. 2018; 154(3):557-66.

11. MacLaren R, Reynolds PM, Allen RR. Histamine-2 receptor antagonists vs proton pump inhibitors on gastrointestinal tract hemorrhage and infectious complications in the intensive care unit. JAMA Intern Med. 2014;174(4): 564-74.

12. Huang HB, Jiang W, Wang CY, Qin HY, Du B. Stress ulcer prophylaxis in intensive care unit patients receiving enteral nutrition: a systematic review and meta-analysis. Crit Care. 2018;22(1):Article 20.

Robert MacLaren, PharmD, MPH, FCCM, FCCP, fait partie de l'équipe de la Skaggs School of Pharmacy and Pharmaceutical Sciences à I'Université du Colorado à Aurora. II est également rédacteur adjoint au Journal canadien de la pharmacie hospitalière.

Conflits d'intérêts : Aucune déclaré

Adresse de correspondance :

$D^{r}$ Robert MacLaren

University of Colorado Skaggs School of Pharmacy

and Pharmaceutical Sciences

12850 East Montview Boulevard, C238

Aurora CO 80045

Courriel : rob.maclaren@cuanschutz.edu 